\title{
Erratum
}

\section{Thermal and non-thermal components of the interstellar medium at sub-kiloparsec scales in galaxies}

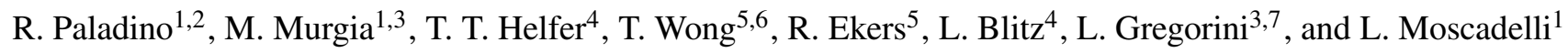 \\ 1 INAF - Osservatorio Astronomico di Cagliari, Loc. Poggio dei Pini, Strada 54, 09012 Capoterra (CA), Italy \\ e-mail: rpaladin@ca.astro.it \\ 2 Dipartimento di Fisica, Università di Cagliari, Cittadella Universitaria, 09042 Monserrato (CA), Italy \\ 3 Istituto di Radioastronomia - INAF, Via Gobetti 101, 40129 Bologna, Italy \\ 4 Radio Astronomy Laboratory, University of California, Berkeley, CA 94720, USA \\ 5 Australia Telescope National Facility, CSIRO, PO Box 76, Epping, N.S.W. 1710, Australia \\ 6 School of Physics, University of New South Wales, Sydney, NSW 2052, Australia \\ 7 Dipartimento di Fisica, Università di Bologna, Via Irnerio 46, 40126 Bologna, Italy
}

Key words. radio continuum: galaxies - galaxies: spiral - ISM: molecules - stars: formation - errata, addenda

Figure 7 published in A\&A, 456 (2006) p. 856 is a duplicate of Fig. 8. Here, we provide the correct version of the figure. The change has no consequences for the discussion in the paper.
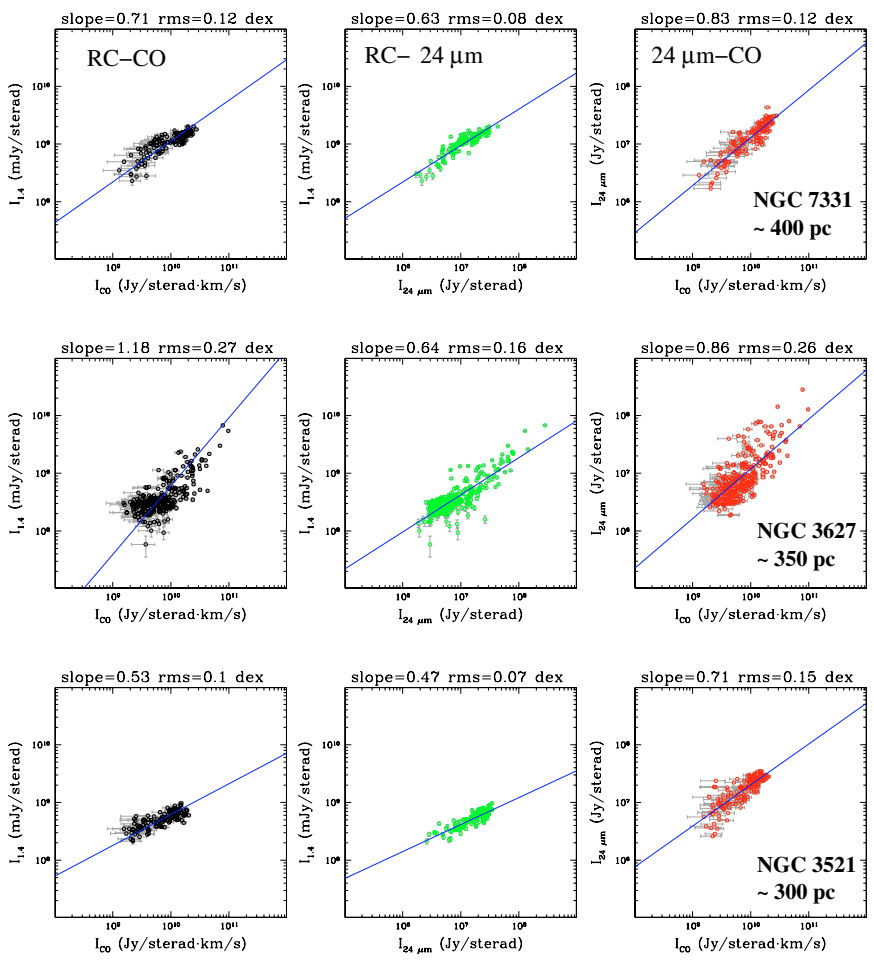

Fig. 1. RC-CO, RC-24 $\mu \mathrm{m}$ and $24 \mu \mathrm{m}$-CO correlations of NGC 7331, NGC 3627 and NGC 3521 at 6 arcsec resolution. The linear resolution achieved for each galaxy is indicated in the lower-right of the right-most panels. 